Con X (N. ${ }^{0}$ 3), e019, octubre 2017

ISSN 2469-0333 | https://doi.org/10.24215/24690333e019

http://perio.unlp.edu.ar/ojs/index.php/conequis

FPyCS | Universidad Nacional de La Plata

La Plata | Buenos Aires | Argentina

\title{
LA APROPIACIÓN DE TÉRMINOS FEMINISTAS COMO NUEVA ESTRATEGIA MEDIÁTICO DISCURSIVA
}

\section{THE APPROPRIATION OF FEMINIST TERMS AS A NEW MEDIA DISCURSIVE STRATEGY}

\author{
Jesica Rodríguez \\ jesicar80@gmail.com \\ http://orcid.org/0000-0001-9346-109X
}

\section{RESUMEN}

En este artículo la autora reflexiona sobre los sentidos sociales que se construyeron y se construyen alrededor de la violencia de género y exponer cómo los medios, a través del uso de un lenguaje que revictimiza y que discrimina a las mujeres, han colaborado y colaboran con el sistema patriarcal vigente. Esto implica remarcar las diferentes estrategias discursivas con que los medios, por medio de distintas categorías lingüísticas, se refieren a la problemática. A su vez, permite advertir continuidades y rupturas discursivas e indagar si es suficiente la inclusión de los términos "femicidios» y «violencia de género» o si se aprecia algún compromiso por erradicar la violencia contra las mujeres.

\section{PALABRAS CLAVE}

medios gráficos, discurso, violencia de género, feminicidio
Investigadora independiente

Argentina

\section{ABSTRACT}

In this article the authoress reflects on the social senses that were constructed and constructed around gender violence and to show how the media, through the use of a language that revictimizes and discriminates against women, have collaborated and collaborated with the current patriarchal system. This implies to remark the different discursive strategies with which the media, through different linguistic categories, refer to the problematic. At the same time, it allows to warn continuities and discursive ruptures and inquiries enough about the inclusion of the terms "femicide" and "gender violence» or if there is any commitment to eradicate violence against women.

\section{KEYWORDS}

graphic media, speech, violence of gender, feminicidio 


\section{LA APROPIACIÓN DE TÉRMINOS FEMINISTAS COMO NUEVA ESTRATEGIA MEDIÁTICO DISCURSIVA}

Por Jésica Rodríguez

El trabajo parte de la hipótesis de que a pese a la incorporación de los conceptos feministas «violencia de género» y «femicidio» en los medios de comunicación los asesinatos de mujeres continúan siendo relatados desde el enfoque del crimen pasional, lo que no permite apreciar un compromiso real de los y las periodistas para erradicar la violencia contra las mujeres.

Desde esta perspectiva, y con el objetivo de entrever los sentidos sociales que se construyeron y que se construyen alrededor de la violencia de género y de explicar cómo los medios a través del uso de determinadas categorías lingüísticas generan su relato sobre la violencia contra las mujeres, se consideró apropiado definir como universo de análisis los feminicidios cometidos por Ricardo Barreda, el 15 noviembre de 1992, y los de La Loma, ocurridos el 26 noviembre de 2011, ambos en la ciudad de La Plata. 
Ambos casos se seleccionaron por la trascendencia que adquirieron para el movimiento de mujeres y feminista, como así también por el alto interés y el impacto mediático que alcanzaron al trascender las fronteras locales. Del mismo modo, porque permiten efectuar un análisis comparado en cuanto al abordaje de la violencia contra las mujeres antes y después de su irrupción mediática, puesto que ocurrieron en distintos momentos históricos, uno en la década del noventa y otro en 2011.

El análisis comparativo permite, además, advertir similitudes y/o diferencias entre uno y otro, señalar rupturas o continuidades discursivas en cuanto a la manera en la que los medios se refieren a la problemática, y observar si el concepto «violencia de género» es utilizado por los y las periodistas. Y, de ser así, de qué modo y en qué contexto resulta empleado.

Por último, se considera acertado inscribir el trabajo en dos contextos diferentes, pero que de alguna manera guardan relación, por un lado, con la situación de los medios de comunicación durante la década del noventa, y, por otro, con el debate contemporáneo sobre las formas que emplean los medios de comunicación para enunciar la violencia que viven las mujeres, y que resurge tras la aplicación de la Ley de Servicios de Comunicación Audiovisual (2009) y debido al aumento de feminicidios, que en la actualidad se cobra la vida de una mujer cada 18 horas. 